\title{
Nonlinear light propagation in fs laser-written waveguide arrays
}

\author{
A. Szameit ${ }^{1}$ and S. Nolte ${ }^{1}$ \\ Institute of Applied Physics, Friedrich-Schiller-Universität Jena, Max-Wien-Platz 1, \\ 07743 Jena, Germany
}

\begin{abstract}
We report on recent achievements in the field of nonlinear light propagation in fs laser-written waveguide lattices. Particular emphasis is thereby given on discrete solitons in such systems.
\end{abstract}

Keywords: discrete systems, nonlinear optics, ultrashort laser pulses, integrated optics

Arrays of evanescently coupled waveguides are a field of wide interest, especially since these structures are a representation of a whole class of physical systems: periodic media. In these media, the light propagation considerably differs from the propagation in a continuous bulk material. In particular nonlinear propagation effects such as discrete spatial solitons attract strong attention, since they exist only in discrete systems and have no stable analogue in continuous media. However, in most cases the experimental demonstration of these phenomena is severely limited due to unrealistically high power thresholds. Recently, it could be shown that the shallow refractive index modulations accessible in waveguide lattices may facilitate the formation of discrete solitons at moderate power levels, in particular at the interface of an array [1]. Due to the discrete nature of waveguide arrays, the light evolves only along the waveguides, while the transverse energy dynamics is based on an evanescent overlap of the individual modes causing a net energy exchange between the individual guides. Hence, the light evolution in such an array can be described by a coupled mode approach, where it is assumed that the transverse shape of the modes remains constant and that only the amplitudes evolve. This results in a set of coupled ordinary differential equations

$$
i \partial_{z} a_{m, n}+\frac{D}{2} \partial_{t t} a_{m, n}+c^{m}\left(a_{m+1, n}+a_{m-1, n}\right)+c^{n}\left(a_{m, n+1}+a_{m, n-1}\right)+\gamma\left|a_{m, n}\right|^{2} a_{m, n}=0,
$$

where $a_{m, n}$ are the propagating amplitudes in the $m, n$th waveguides ( $m, n$ account for the transverse directions) and $z$ is the coordinate in propagation direction. The value $\gamma$ specifies the nonlinear cubic response of the material while the strength of the evanescent coupling between the individual guides is characterized by the coupling coefficients $c^{m}$ and $c^{n}$. The quantity D characterizes the group velocity dispersion and vanishes for purely spatial dynamics (i.e., cw beams). For the investigation of spatial nonlinear effects, which counterbalance the linear diffraction, usually a very homogeneous coupling between the single lattice sites is required, allowing a precise modelling of the system's response. However, with conventional fabrication techniques like optical induction in photorefractives and waveguide arrays in fibers, it is hard to meet this requirement.

Recently it was discovered that one can use tightly focused fs laser pulses to write waveguides directly into transparent bulk materials [2]. In the focal volume of the focusing objective very high energy densities yield multiphoton absorption and the formation of electron plasma. This results in a permanent change of the material's molecular structure which can, under appropriate conditions, lead to an increase of the refractive index. This approach exhibits several strong advantages: First, the refractive index modifications are stable and do not decay over years. Second, the waveguide can be written along almost arbitrary paths. And third, the waveguides are written one by one, so that the individual properties of every guide, such as position, strength and nonlinearity, can be specifically tuned. This allows in particular the realization of sharply defined array boundaries and interfaces [3].

We report on our recent achievements in the experimental investigation of discrete solitons in fs-laser written waveguide arrays in fused silica. Typical sample parameters are a length of $100 \mathrm{~mm}$ and a waveguide separation of 35 $\mu \mathrm{m}$. Since the nonlinearity in the waveguides is purely electronic, for the excitation of the solitons very high amplitudes are required, which can only be generated using ultrashort laser pulses. In our experiments we launch pulses at $800 \mathrm{~nm}$ with a length of $100 \mathrm{fs}$ into a single waveguide, so that for a peak power much larger than $1 \mathrm{MW}$ the formation of discrete solitons with one [4] and two [5] transverse dimensions can be demonstrated (Fig. 1a,b).

Whereas nonlinearity can compensate diffraction, certain linear wave packets with specific shapes are known to propagate without changing their shape. Beside the well-known spatial Bessel beams, spatio-temporal X-waves are the most prominent examples of nondiffracting waves. Despite being an inherently linear phenomenon, X-waves can also 
emerge from the decay of a nonlinearly propagating pulse. A necessary precondition for the existence of X-waves are opposite signs of diffraction and dispersion, corresponding to X- or cone-shaped iso-surfaces of the dispersion relation [see Figure 1(c) for a square lattice]. Our detailed investigations of the spatiotemporal dynamics during pulse propagation through a photonic lattice with square geometry lead to the first realization of X-waves in two transverse dimensions [6]. Their characteristic shape [see Figure 1(d)] emerges spontaneously from a single-site excitation due to the interplay of Kerr nonlinearity, discrete diffraction and dispersion. Rigorous numerical simulations confirmed the experimental results and indicated the formation of cascaded X-waves for longer propagation distances. Although these observations show that spatiotemporal effects are non-negligible, the conical emissions from the slopes merely introduce a certain diffractive background, while the spatial distribution of light at the sample end face is still dominated by the well localized pulse core. Consequently, dynamic excitation yields qualitatively similar results that allow the investigation of spatial solitons.

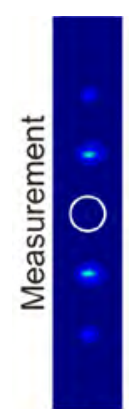

(a)

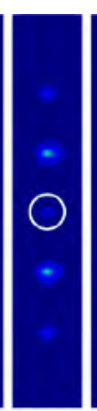

0.3
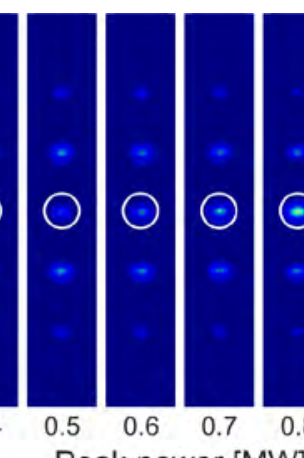

Peak power [MW]

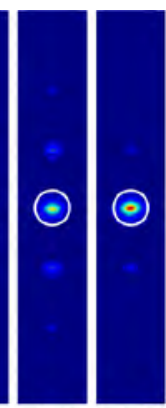

$0.9 \quad 1.0$
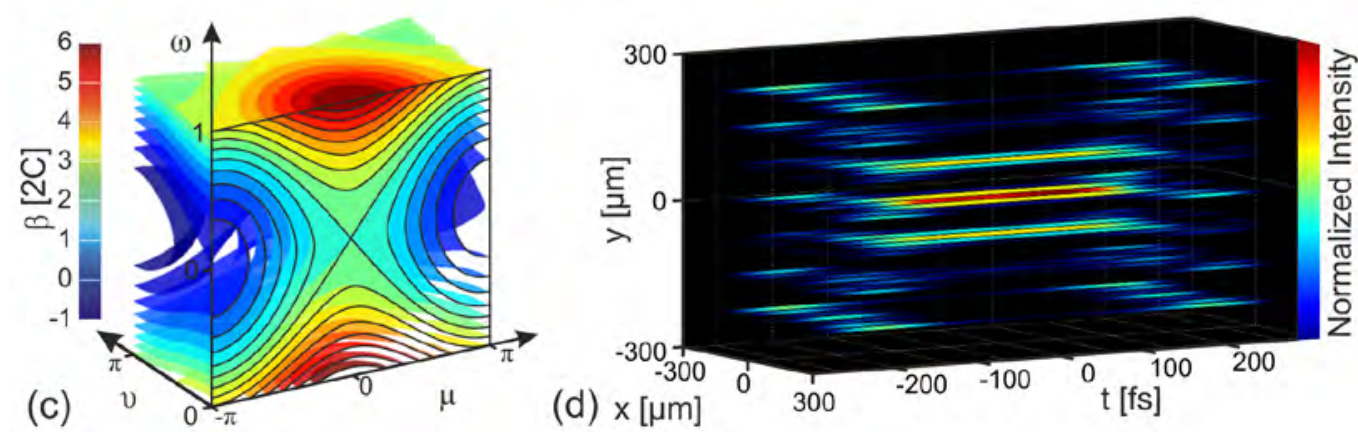

(Fig. 1) Upper row: discrete spatial soliton formation. (a) Soliton formation in a planar lattice [4]. Measured diffraction patterns at various peak powers. (b) Soliton formation in a 2D lattice with square geometry [5]. Measured [top] and simulated [bottom] diffraction patterns at various input peak powers. The excited lattice sites are marked with white circles.

Lower row: Observation of three-dimensional discrete-continuous X-waves in photonic lattices [6]. (c) Dispersion relation $\beta(\mu, v, \omega)$ of a square lattice. (d) Spatiotemporal pulse shape after nonlinear propagation of a single-site excitation through the sample.

Future research directions will involve various degrees of freedom of the fs laser direct writing technique that have been omitted so far. This includes besides the direct shaping of the structural modification the fabrication, characterization and utilization of nanogratings, which brings forward the idea of a tunable birefringence in integrated optical devices [7]. Additionally, due to the ability to directly influence the nonlinearity of the structured medium, nonlinearity itself may become a free parameter that can be tuned at will. A further direction will also be the experimental generation and analysis of purely nonlinear spatio-temporal phenomena, as the recently observed discrete-continuous spatial-temporal light bullets [8]. And last but not least, all the experiments so far were performed in fused silica as the bulk material. However, the fs laser writing technique is versatile and can be applied to various materials apart from fused silica, such as e.g. Lithium Niobate [9].

[1] F. Lederer et al., Phys. Rep. 463, 1 (2008).

[2] K. Davis et al., Opt. Lett. 21, 1729 (1996).

[3] M. Heinrich et al., Appl. Phys. B 104, 469 (2011).

[4] A. Szameit et al., Opt. Exp. 13, 10552 (2005).

[5] A. Szameit et al., Opt. Exp. 14, 6055 (2006).

[6] M. Heinrich et al., Phys. Rev. Lett. 103, 113903 (2009).

[7] L. P. R. Ramirez et al., Appl. Phys. A. 100, 1 (2010).

[8] S. Minardi et al., Phys. Rev. Lett. 105, 263901 (2010).

[9] J. Thomas et al., Appl. Phys. Lett. 91, 151108 (2007) 